\title{
Review of: "Characteristics, Decoupling Effect And Driving Factors of Regional Tourism's Carbon Emissions In China"
}

\author{
Kaifeng Wang ${ }^{1}$ \\ 1 South China Normal University
}

Potential competing interests: The author(s) declared that no potential competing interests exist.

Thank you for inviting me to review this paper, which lies close to my research interest.

In general, this manuscript shows a decoupling analysis process of carbon emissions, which has impressed me deeply. However, to improve the quality of this study, some necessary revisions are still needed:

1. Introduction

\section{Literature Review}

line 53: The number of references is insufficient, and the literature is not organized according to the content framework or theoretical logic of this manuscript. Half of the literature here is on carbon emission measurement techniques, however, this is only a basic method used in this manuscript.

line 64: The second paragraph of the literature review section starts with "In terms of research content", which is not a good enough literature review idea, and the two studies that follow are actually spatial analysis, which is not related to this manuscript closely. A possible path for improvement is that the authors should expand the literature reference to describe the use of the two main research methods in this manuscript in the existing literature in the same field, as well as the findings and conclusions obtained by the researchers.

line 78: The authors do not actually have an accurate understanding of the innovative or marginal contribution of their manuscripts, because I can find that the authors do not adequately describe the merits or innovativeness of their research, just by referring to the following three references:

ZHANG, G. H., \& LIU, J. (2015). Analysis on Decoupling Relationship Between Tourism Development and Carbon Emission in Coastal Region of China. Resource Development \& Market, 11.

Tang, Z., Bai, S., Shi, C., Liu, L., \& Li, X. (2018). Tourism-related CO2 emission and its decoupling effects in China: a spatiotemporal perspective. Advances in Meteorology, 2018.

Chen, L., Thapa, B., \& Yan, W. (2018). The relationship between tourism, carbon dioxide emissions, and economic growth in the Yangtze River Delta, China. Sustainability, 10(7), 2118.

\section{Methods and Data}


lines 115-116: There are many formatting errors in the manuscript, for example, the citations here are marked incorrectly: (Nae-Wen Kuo et al. 2009; Shi Peihua et al. 2011). Authors should exam the entire manuscript to avoid formatting errors like this.

line 124: The authors should explain whether the method of accounting for carbon emissions is consistent with IPCC standards.

line 158: The impact factor analysis of this manuscript completely relies on the LDMI method, which is actually mathematical statistics rather than empirical testing, which is not conducive to the scientific nature of the research work in this manuscript. Therefore, econometrics methods are suggested to be supplemented in this manuscript.

4. Results

5. Conclusions and Implications 